\section{Off-label use of inhaled tobramycin in Ontario, Canada}

Inhaled tobramycin solution is indicated for use in the management of Pseudomonas aeruginosa in patients with cystic fibrosis (CF). Concerns have been raised regarding increasing off-label use of inhaled tobramycin, particularly for the management of COPD. We conducted an 8-year repeated cross-sectional study examining the indication for prescription claims for inhaled tobramycin in Ontario paid for by the Public Drug Benefit Program, which covers all Ontario residents with financial needs or aged 65 and older. Inhaled tobramycin prescription claims increased approximately 3 times greater from 86 prescriptions in the second quarter of 2007 to 261 prescriptions in the first quarter of 2015. Approximately half of all prescriptions (range: 46-65\%) per quarter were dispensed to patients with CF. A large proportion of prescriptions (range: 31-36\%) were dispensed to individuals who did not have a diagnosis of CF but had a diagnosis of COPD. In 2014, there were 324 unique users of inhaled tobramycin solution in the Ontario Public Drug Program (OPDP). Only half of users ( $54 \% ; n=163)$ had a diagnosis of CF. Our study found increasing prescriptions of inhaled tobramycin from 2007 to 2015 in the OPDP with approximately half of these claims being for off-label use, mostly among patients with COPD.

\section{BACKGROUND}

Inhaled tobramycin solution was approved in Canada in 1999 and is indicated for the management of Pseudomonas aeruginosa in patients with cystic fibrosis (CF). Inhaled tobramycin has been shown to improve lung function and quality of life in patients with CF. ${ }^{12}$ Since its introduction, tobramycin has been marketed for other off-label indications, leading to concern regarding its increasing off-label use. ${ }^{3}$ There is little evidence to support the off-label use of tobramycin, but early work has suggested possible benefits in patients diagnosed with COPD. ${ }^{4}$ Inhaled tobramycin was added to the Ontario Drug Benefit Formulary in 2006 as a general benefit intended for use in patients with CF. The objective of our study was to examine changes in the apparent indication of inhaled tobramycin use over time.

\section{METHODS}

We conducted a repeated cross-sectional study examining quarterly prescription claims for inhaled tobramycin reimbursed by the Ontario Public Drug Program (OPDP) from April 2007 to March 2015. This programme is available for all those with financial needs (due to high drug costs and/or low income) and all residents 65 years of age and older. Prescription claims for patients who receive medications through private coverage or pay out of pocket are not captured in this database. The total number of prescriptions and users were reported for each quarter and stratified by those with a diagnosis of CF or COPD. CF diagnosis was based on a look back of 5 years from the date of the prescription for International Classification of Diseases-10 (ICD-10) code E84 in emergency department visits or inpatient hospital admissions, or for diagnostic code 277 in Ontario Health Insurance Plan (OHIP) physician claims. Sensitivity of the codes for detecting CF diagnosis was assessed by comparing those in an older CF Registry with those located with this algorithm to ensure a high level of agreement. ${ }^{5}$ COPD diagnosis was based on diagnosis in a validated COPD cohort with a diagnosis date prior to the prescription. ${ }^{6}$ Individuals with both CF and COPD were categorised as CF to allow two mutually exclusive groups; patients with neither diagnosis were also excluded. Further, we examined individuals who had received at least one prescription for inhaled tobramycin in calendar year 2014 to describe differences in age and tobramycin utilisation among patients with $\mathrm{CF}$ compared with patients diagnosed with COPD. We stratified users by age $(<65$ or $65+)$ and those who received only one prescription for tobramycin.

All drug claims were identified using the Ontario Drug Benefit Database from the Ontario Drug Benefit Program, which contains all prescriptions dispensed to eligible Ontario residents. Prescription claims for patients who receive medication through private drug coverage or pay out of pocket were not captured. All prescriptions were based on a prescription claim. A user may have more than one claim in a quarter. Under the Ontario Drug Benefit Program, the initial claim for a medication cannot exceed a month's supply of drugs and further claims cannot exceed 100 days. We identified hospital admissions data from the Canadian Institute for Health Information Discharge Abstract Database and physician billing data from the OHIP Claims History Database. These databases are linked by encrypted health card numbers and are routinely used in drug policy research. ${ }^{78}$

\section{RESULTS}

Over the 8-year study period, inhaled tobramycin claims increased approximately 3 times greater from 86 in the second quarter (Q2) of 2007 to 261 in the first quarter (Q1) of 2015, figure 1. Approximately half of all prescriptions $(52 \%, n=3042$ of 5853 ; range: $46-65 \%)$ between Q2 2007 and Q1 2015 per quarter over the study period were dispensed to patients with CF. A large proportion of prescriptions $(40 \% ; n=2350$; range: $31-36 \%$ ) were dispensed to individuals who did not have a diagnosis of $\mathrm{CF}$ but had a diagnosis of COPD. A small proportion of prescriptions $(7.8 \%$; $\mathrm{n}=461$ ) were dispensed to individuals who did not have a diagnosis of either CF or COPD. In the same time period, the number of users increased at the same rate from 53 in the second quarter $(\mathrm{Q} 2)$ of 2007 to 173 in the first quarter (Q1) of 2015, online supplement.

In 2014, there were 324 unique users of inhaled tobramycin solution in the OPDP. Of these users, 300 users had a known diagnosis of either CF or COPD, table 1. One-third of these users $(32 \%$; $\mathrm{n}=95$ ) received only one prescription. Half of users $(54 \% ; n=163)$ had a diagnosis of CF; 46\% ( $n=137)$ did not have a CF diagnosis, but did have a diagnosis of COPD. Over half $(52 \% ; n=157)$ of inhaled tobramycin users were under the age of 65 , and the prevalence of $\mathrm{CF}$ and COPD differed considerably by age. Of those with CF, only 15 (9\%) were over the age of 65 . Of those with a COPD diagnosis, the vast majority (93\%; $n=128$ ) were over the age of 65 .

\section{DISCUSSION}

We found increasing utilisation of inhaled tobramycin in the OPDP over the 8-year window examined. There was evidence of considerable off-label use over the 8 years. Approximately half $(48 \%)$ of all inhaled tobramycin utilisation was apparently off-label.

A large proportion (40\%) of the apparent off-label use was in patients with COPD. Although there is current inquiry into the possible benefit of these agents for off-label indications, these indications are presently not supported by robust clinical trials. ${ }^{4}$ Indications such as bronchiectasis, ventilator-associated pneumonia, post-transplant airway infections and drug-resistant infections have also been suggested in the literature. ${ }^{4}{ }^{10}$ Safety of inhaled tobramycin in off-label populations is also unknown, with specific concern of nephrotoxicity in patients with COPD. ${ }^{11}{ }^{12}$ Further exploration of the effectiveness and safety is necessary in these off-label indications as their use can be anticipated to grow as use of inhaled tobramycin also continues to increase. ${ }^{13}$ 


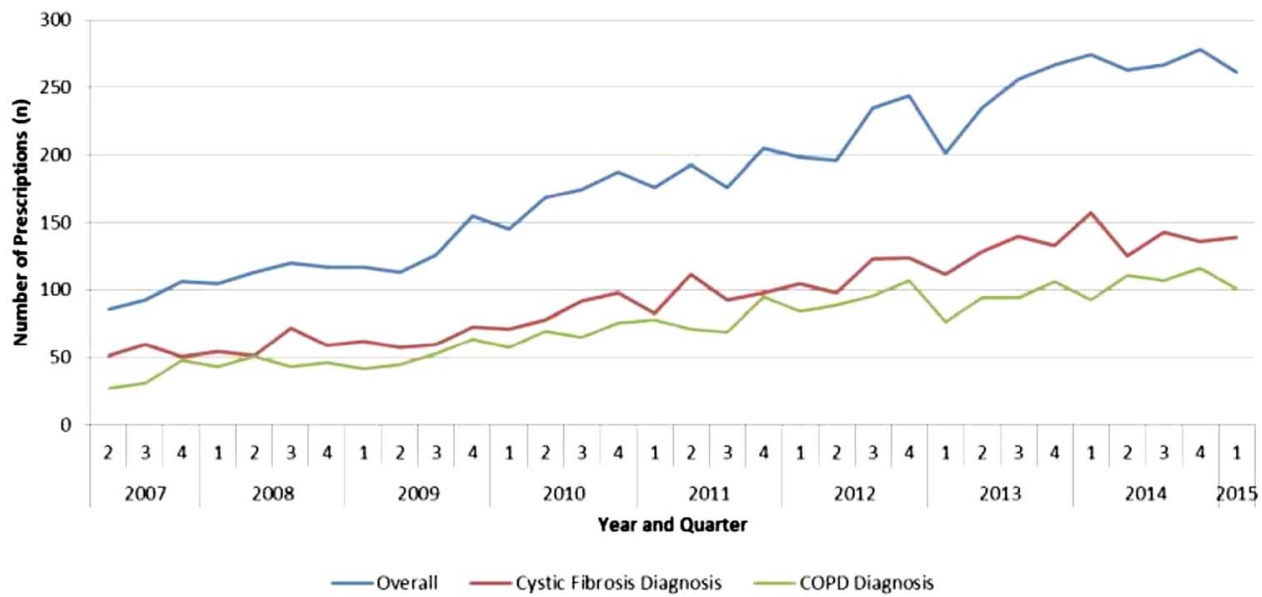

Figure 1 The use of inhaled tobramycin solution in the Ontario Public Drug Program, between Q2 2007 and Q1 2015. Patients with COPD did not have a diagnosis of cystic fibrosis.

A high proportion $(32 \%)$ of users were found to have only one prescription in both patients with CF (36\%) and patients with COPD (27\%); future work should explore trends in persistence and discontinuation. Additionally, the prevalence of use of these products differs among CF and COPD populations. In 2014, there were a projected 1639 patients with CF compared with 932624 patients with COPD in Ontario, an estimated 9.9\% and $0.01 \%$ prevalence of inhaled tobramycin use, respectively. These results are important to payers as they assess the listing and reimbursement of these products on their formularies. These findings may warrant stricter restrictions of reimbursement to only patients with $\mathrm{CF}$ and only lead to expansion to patients with COPD upon development of stronger evidence. This type of drug utilisation research has been shown to be useful to policymakers and payers. ${ }^{9}$ Previous drug utilisation studies have led to policy recommendations of expansion or restrictions of formulary listing of drugs.

Our study has some limitations. We used claims data and thus are not certain of the actual indication and dosing of inhaled tobramycin. To strengthen the confidence in the codes used for CF diagnosis, we replicated the analysis using a CF Registry for a small portion of the study period where data were available and found consistent results. ${ }^{5}$ Second, we leveraged a validated database to identify COPD diagnoses; ${ }^{6}$ however, for patients without CF or COPD, we are not certain of the indications of use. We were unable to explore which patients had COPD with or without bronchiectasis. This should be further explored in future work as it has been recommended that those with bronchiectasis may benefit from inhaled tobramycin. ${ }^{10}$ Lastly, our study does not include prescription claims paid for by private insurers or out of pocket by patients. Our results are representative of the claims paid for by the OPDP and thus may not be generalisable to all payers.

After the listing of inhaled tobramycin on the Ontario Public Drug Formulary in 2006, its use has increased considerably, and only half of prescriptions were prescribed to individuals with a diagnosis of CF. This suggests that there is a large amount of off-label use in patients with COPD, where there is little evidence of efficacy from clinical trials. Given the potential implications of off-label

Table 1 Inhaled tobramycin solution users in the Ontario Public Drug Program, by indication, between 1 January 2014 and 31 December 2014†

\begin{tabular}{llll}
\hline & $\begin{array}{l}\text { Total } \\
\text { N, (\%) }\end{array}$ & $\begin{array}{l}\text { Users with cystic } \\
\text { fibrosis (CF) }\end{array}$ & $\begin{array}{l}\text { Users without CF } \\
\text { with COPD }\end{array}$ \\
\hline Number of users & $\mathrm{N}=300$ & $\mathrm{~N}=163(54 \%)^{*}$ & $\mathrm{~N}=137(46 \%)^{*}$ \\
Age (median, IQR) & $57(21-76)$ & $23(13-32)$ & $76(71-81)$ \\
Number with only one prescription N (\%) & $95(32 \%)$ & $58(36 \%)$ & $37(27 \%)$ \\
Age 65+ & $143(48 \%)$ & $15(9 \%)$ & $128(93 \%)$ \\
\hline
\end{tabular}

*The per cent of total number of users in the corresponding row.

tThe results were limited to only those with a known indication of CF or COPD. Users with no known indication are not shown.

Thorax September 2016 Vol 71 No 9

tobramycin use on both drug costs and patient outcomes, future research should explore the effectiveness and safety of inhaled tobramycin in these off-label indications, especially in patients with COPD.

\section{Mina Tadrous, ${ }^{1,2,3}$ Wayne Khuu, ${ }^{2}$ \\ J Michael Paterson, 2,4,5,6 \\ Muhammad M Mamdani, ${ }^{1,2,3,4,7,8}$ \\ David N Juurlink, ${ }^{2,4,8,9,10}$ Tara Gomes ${ }^{1,2,3}$}

${ }^{1}$ St. Michael's Hospital, Toronto, Ontario, Canada ${ }^{2}$ Institute for Clinical Evaluative Sciences, Toronto, Ontario, Canada

${ }^{3}$ The Leslie Dan Faculty of Pharmacy, University of Toronto, Toronto, Ontario, Canada

${ }^{4}$ Health Policy, Management, Evaluation, University of Toronto, Toronto, Ontario, Canada

${ }^{5}$ Department of Family Medicine, McMaster University, Hamilton, Ontario, Canada

${ }^{6}$ Centre for Evaluation of Medicines, St. Joseph's Healthcare, Hamilton, Ontario, Canada

${ }^{7}$ Department of Medicine, St. Michael's Hospital, Toronto, Ontario, Canada

${ }^{8}$ Department of Medicine, University of Toronto, Toronto, Ontario, Canada

${ }^{9}$ Sunnybrook Research Institute, Toronto, Ontario, Canada

${ }^{10}$ Department of Pediatrics, University of Toronto, Toronto, Ontario, Canada

Correspondence to Dr Mina Tadrous, St. Michael's Hospital, 30 Bond St, Toronto, Ontario, Canada M5B 1W8; tadrousm@smh.ca

Twitter Follow Mina Tadrous at @mina_t

Contributors All authors were involved in the design, interpretation of results, writing, conceptualisation of recommendations and revision of the manuscript. MT, MK and TG were involved in the implementation of the study.

Funding This study was funded by a grant from the Ontario Ministry of Health and Long-Term Care (MOHLTC) Health System Research Fund and supported by the Institute for Clinical Evaluative Sciences (ICES), a non-profit research institute sponsored by the Ontario MOHLTC. The opinions, results and conclusions reported in this paper are those of the authors and are independent from the funding sources. No endorsement by ICES or the Ontario MOHLTC is intended or should be inferred. Parts of this material are based on data and information compiled and provided by Canadian Institute for Health Information (CIH)). However, the analyses, 


\section{Research letter}

conclusions, opinions and statements expressed herein are those of the authors and not necessarily those of CIHI. We thank Brogan Inc, Ottawa, for use of their Drug Product and Therapeutic Class Database.

Competing interests MMM has received honoraria from Boehringer Ingelheim, Pfizer, Bristol-Myers Squibb and Bayer.

Ethics approval This project was approved by the research ethics board of Sunnybrook Health Sciences Centre, Toronto.

Provenance and peer review Not commissioned; externally peer reviewed.

- Additional material is published online only. To view please visit the journal online (http://dx.doi.org/10. 1136/thoraxjnl-2015-208145).

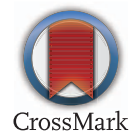

To cite Tadrous M, Khuu W, Paterson JM, et al. Thorax 2016;71:862-864.

Received 30 November 2015

Revised 14 April 2016

Accepted 15 April 2016

Published Online First 10 May 2016
Thorax 2016;71:862-864.

doi:10.1136/thoraxjnl-2015-208145

\section{REFERENCES}

1 Mogayzel PJ Jr, Naureckas ET, Robinson KA, et al. Cystic fibrosis pulmonary guidelines: chronic medications for maintenance of lung health. Am J Respir Crit Care Med 2013;187:680-9.

2 Ramsey BW, Pepe MS, Quan JM, et al. Intermittent administration of inhaled tobramycin in patients with cystic fibrosis. N Engl J Med 1999;340:23-30.

3 Novartis vaccines \& diagnostics to pay more than $\$ 72$ million to resolve False Claims Act allegations concerning TOBI. Secondary Novartis vaccines \& diagnostics to pay more than $\$ 72$ million to resolve False Claims Act allegations concerning TOBI 2010. http://www.justice.gov/opa/pr/novartis-vaccinesdiagnostics-paymore-72-million-resolve-false-claims-act-allegations

4 Quon BS, Goss CH, Ramsey BW. Inhaled antibiotics for lower airway infections. Ann Am Thorac Soc 2014;11:425-34.

5 Stephenson A. Predictors of hospitilization among cystic fribrosis patients in Ontario. University of Toronto, 2010.

6 Gershon AS, Wang C, Guan J, et al. Identifying individuals with physician diagnosed COPD in health administrative databases. COPD 2009;6:388-94.
7 Mamdani M, Juurlink DN, Kopp A, et al. Gastrointestinal bleeding after the introduction of COX 2 inhibitors: ecological study. BMJ 2004;328:1415-16.

8 Gomes T, Mamdani MM, Dhalla IA, et al. Opioid dose and drug-related mortality in patients with nonmalignant pain. Arch Intern Med 2011;171:686-91.

9 Khan S, Moore JE, Gomes T, et al. The Ontario Drug Policy Research Network: bridging the gap between research and drug policy. Health Policy 2014:117:392-8.

10 Yang JW, Fan LC, Lu HW, et al. Efficacy and safety of long-term inhaled antibiotic for patients with noncystic fibrosis bronchiectasis: a meta-analysis. Clin Respir J Published Online First: 26 Jan 2015. doi: $10.1111 /$ cri. 12278

11 Cannella CA, Wilkinson ST. Acute renal failure associated with inhaled tobramycin. Am J Health Syst Pharm 2006;63:1858-61.

12 Izquierdo MJ, Gomez-Alamillo C, Ortiz F, et al. Acute renal failure associated with use of inhaled tobramycin for treatment of chronic airway colonization with Pseudomonas aeruginosa. Clin Nephrol 2006;66:464-7.

13 Pasteur MC, Bilton D, Hill AT. British Thoracic Society guideline for non-CF bronchiectasis. Thorax2010;65 1):i1-58. 